BIRGER P. PRIDDAT

\title{
eGovernment/eDemocracy: Eine neue Dimension der Gemeinwohlermittlung in der Politik
}

\section{1. eDemocracy}

Die in der Mitte der 90er Jahre begonnene Diskussion um die, electronic democray ${ }^{4}$ ist längst kein utopisches Projekt mehr, wie es die erregt diskutierte ,Teledemokratie' der 70er Jahren noch war. ${ }^{1}$ Sie stellt nicht die Wahlverfahren auf Knopfdruck um, sondern eröffnet völlig neue Informations- und Beteiligungslandschaften über das Internet: Netzwerkinteraktionen. ${ }^{2}$ Der Topos der Kommunikation, der für die politische Ökonomie entdeckt wird, erstreckt sich heute über den medialen Diskurs der Öffentlichkeit hinaus auf ein Kommunikationsmedium, dessen eigenständige Valenz hervorsticht: das Internet. Kommunikation ist nicht mehr nur Diskurs und Öffentlichkeit, sondern auf eine informationstechnologische Struktur bezogen, die eigene, multiple und diversifizierte Öffentlichkeiten herstellt. Die, Macht der Medien ' wird durch Medien eigener Macht komplettiert.

Es geht nicht um anonyme Einspeisung von Wahlentscheidungen auf technisch neue Art, sondern um Informations- und Transparenzdimensionen zur Entscheidungsfindung, wie sie zuvor nicht möglich schienen. Internet-communities können untereinander kommunizieren, was in welcher Form gestaltet werden soll. Das Internet schafft nach dieser Vorstellung policy networks, Kommunikationsarenen, oder: eGoras (electronic agoras).

I Vgl. Zolo 1998, S. 204; Hagen 1997.

2 Vgl. für die Entwicklung in Deutschland Stradtmann 1998.

3 Wenn auch das wieder kritisch gesehen wird, z.B. Sassen 2000; Schmalz-Bruns 2000. Dabei ist nicht zu befürchten, daß die klassischen Medien (Print, TV) außer Kraft gesetzt werden. Sie bleiben notwendig komplementär bestehen, weil das Internet sortierende und orientiere Medien braucht. Das Internet thematisiert nichts selber, sondern nur die Nutzer, die sich auf bestimmte Portale massiv einschwenken. Presse und Fernsehen werden neue Bedeutung bekommen als Leitmedien der thematischen Fokussierung des Internetüberangebotes an Themen und Thematisierungen. Beide Medienarten schaukeln sich wechselseitig hoch. Das Internet liefert den Themenüberschuß, TV und Zeitungen sortieren und orientieren. 
Dennoch ist diese Form der eDemocracy nur ein Aspekt des neuen vernetzten Politikparadigmas. Benjamin R. Barber unterscheidet drei Formen der Demokratie:

1. die ,thin" oder "representative democracy“,

2. die ,plebiscitary democracy" und

3. die , strong demacracy". ${ }^{4}$

Die repräsentative Demokratie erzeugt relativ passive Bürger, sogenannte, monitors $^{6}{ }^{5}$ $\mathrm{Zu}$ den plebiszitären Modellen notiert Barber, „few would argue that the plebiscitary model is anything other than a corruption of deliberative democracy ". ${ }^{6}$ Barber hebt die „strong democracy“ als die geeignete Form im Informationszeitalter hervor: „In strong democracy, citizens actually participate in governing themselves, if not in all matters, all of the time, at least in some matters at least some of the time. ${ }^{.7}$ Da die digitalisierten Medien auf Geschwindigkeit abonniert sind, schlägt Barber für die, strong democracy vor, die „multi-reading referendum procedure“ über sechs Monate zu fahren - „slow down digital media". ${ }^{8}$

Barbers Plädoyer für die Demokratie als reflexive Instanz steht gegen Bill Clintons Versprechen, das eGovernment als ",high-speed, high-tech, user-friendly government" einzuführen. ${ }^{9}$ Die Spannung zwischen Geschwindigkeit und Reflexivität (Diskursdauer als Diskursqualität) bestimmt fortan die Formen der Organisation von der Politik, in denen sich differente Entscheidungsarenen ausbilden werden, die auf die verschiedenen Geschwindigkeitsanforderungen unterschiedlich reagieren und antworten.

Hier wird ein Thema angesprochen, das für den Wandel der Formen der Demokratie nicht nur unter dem Gesichtspunkt von e-democracy bedeutsam ist:

- Demokratie wird partizipativer verstanden, als Einbringen von Präferenzen und Erfahrungen (Wissen) der Bürger in Entscheidungszusammenhänge.

- Zugleich wird eine neue Legitimationsebene entfaltet: die Formen direkter Demokratie dort einzuführen, wo die Bürger als kompetent gelten, d.h. nicht auf allen Ebenen.

Das verstößt anscheinend gegen das egalitäre Demokratieprinzip, alle Bürger mit entscheiden zu lassen in allen Fragen, die sie angehen. Dieses Prinzip bricht aber bereits dort ab, wo es um kommunale, lokale Fragen geht; die Bürger von Nachbarkommunen geht das nichts an. Unterhalb des formalen Demokratieprinzips wählen und entscheiden sowieso nur die Bürger, die faktisch wählen gehen, also niemals alle. Formal fordert Demokratie die Option der Wahl für alle Bürger, nicht die Pflicht. Barbers Hinweis ist insofern neu, als er Politik nicht nur als Resultat von Engagement

4 Barber 1998, S. 4.

5 Ebd. Der Term ,monitors` kommt von Schudson 1998, der ihn positiv, als ultima ratio des democratic citizen verwendet.

6 Barber 1998, S. 5.

7 Ebd.

8 Ebd., S. 7.

9 The White House, Office of the Press Secretary, June, 24, 2000; Remarks By The President In First Internet Webcast. 
und Thematisierung beschreibt, d.h. als einen keimhaft sich selber organisierenden Prozeß, der alle, die es angeht, hineinzunehmen anbietet, sondern daß er auf die Zeitcharakteristik dieses Prozesses, auf seine Dauer eingeht. Nicht die Dauer ist entscheidend, sondern daß ein Prozeßraum eröffnet wird, innerhalb dessen eine langsam sich aufbauende und modifizierende Thematisierung stattfindet, die die Eingangspräferenzen ändert. ${ }^{10}$

Der Politikprozeß wird als ein Präferenzgenese- und Änderungsprozeß eingeführt; hier beginnen neue Qualitäten, die nicht als Frage-Antwort-Abstimmungsspiel laufen, sondern als inszenierter Diskurs. eDemocracy bekommt über das Internet als policy-net eine Diskursmateriatur neuer Qualität. Burt Mulders z.B. entwirft eine postparlamentarische Form der Demokratie, in der die politischen Entscheidungen dezentriert und die parlamentarische Willensbildung auf eine Öffentlichkeits- und Allokationsfunktion reflexiv zugeschnitten wird. ${ }^{11}$

Die euphorischen Phantasien der digitalen Durchdemokratisierung moderner Gesellschaften - cyber-democracy - werden inzwischen selbst in den USA vorsichtiger und nüchterner betrachtet, ${ }^{12}$ in Deutschland sowieso. ${ }^{13}$ Der Kern der Möglichkeiten von eDemocracy schält sich heraus. eDemocracy ist eine neue Variante von direkter Demokratie. Direkte Demokratien sind Instanzen des Monitoring der Politik durch die Bürger selber. Wir haben es mit Projekten der Regulation der Regulation zu tun. Dabei spielen oft überzogene Steuerungsphantasien eine Rolle. Funktional betrachtet arbeitet die Demokratie wie folgt:

1. Bürger wählen Politiker/Parteien/Programme.

2. Die Wahlsieger bilden Regierungen.

3. Regierungen machen Politik.

Nun ist das Politikergebnis oft nicht konform mit dem, weswegen die Regierungen gewählt wurden. Politik enttäuscht, und zwar aus systematischem Grund: Der Politikprozeß, d.h. die Wahl der Politikstrategien und die Durchsetzung von Politik, spielt sich in einer Matrix vielfältiger Bedingungen und Einflüsse ab, die alle vermittelt und selegiert werden müssen. Die ursprünglichen Wählerpräferenzen werden in diesem Politikprozeß durch andere Interessen, z.B. die der Politiker, wiedergewählt zu werden, modifiziert, aber auch der Realismus der Situation schlägt zu, Einflußgruppen und Lobbies spielen herein, außenpolitische Rücksichtnahmen etc. Wählerpräferenzen sind bruttoPräferenzen, deren politischer Ertrag netto ausgezahlt wird, d.h. durch Restriktionen gefiltert und modifiziert, die nicht Element der ursprünglichen Präferenz waren - und auch nicht sein konnten, weil die effektiven Limitationen des politischen Prozesses, der nach den Wahlen einsetzt, ex ante nicht bekannt sind. Die Antizipation der Möglichkeit dieser Restriktionen wird wählerseitig als Inkompetenzvermutung gegenüber Politikern geäußert. Deshalb ist folgende zusätzliche Unterscheidung des gesamten Demokratieverfahrens einzuführen:

${ }^{10} \mathrm{Vgl}$. Slaton 1998 und Fishkin 1998.

11 Vgl. Mulder 1999, S. 560-564.

12 Norris 2000

$13 \mathrm{Vgl}$. Schmalz-Bruns 2000. 
I. Wahlprozeß (elections);

II. Politikprozeß (post electional process).

Zum Wahlprozeß (I.) gehören 1. und 2., zum Politikprozeß (II.) gehört 3. Es wäre unzulässig, eDemocracy einfach nur als Digitalisierung des Wahlprozesses (I.) zu sehen. Die entscheidende Innovation ist die Integration der Bürger in den Politikprozeß (II.): post electional process.

Einflüsse und Mitentscheidungen - wie in den korporatistischen Arrangements des ,Bündnis für Arbeit' etc., aber auch bilaterale Formen der Interessenvertretung durch lobbying - bilden eine $2^{\text {nd }}$-order-democracy, d.h. eine zweite Eintragsebene von Wählerpräferenzen.

Nur haben zu dieser Ebene nicht alle Bürger Zugriff. Hier spielen vornehmlich organisierte Interessen hinein. Die Demokratie zweiter Ordnung arbeitet im Politikprozeß (II.), nicht im Wahlprozeß (I.). Hier ist die eDemocracy neu zu adjustieren.

eDemocracy läßt sich sinnvoll als Extension der Demokratie zweiter Ordnung neu konfigurieren: als durch das Internet ermöglichte Ausweitung der Planungs- und Entscheidungshereinnahme von Bürgern in den Politik-, nicht nur in den Wahlprozeß. Damit verzahnt sich eDemocracy eng mit dem eGovernment, wie wir noch entfalten werden, d.h. mit den durch das Internet möglichen neuen Steuerungskonzeptionen. eDemocracy ist keine technisch neue Form des Wählens, sondern wird selber ein Element einer neuen Politik, die die Bürger stärker als bisher in den Politikprozeß hineinnimmt. Es geht darum, die Politik in die Politik zurückzuholen.

eDemocracy wird eine Form der Wissenskommunikation, die der Staat für seine Steuerung benötigt, um erfolgreich zu bleiben. Er kommuniziert mit den Bürgern die Politik, nicht nur im, Wahlkampf", sondern im laufenden Politikprozeß.

\section{Digital divide}

Pippa Norris hat die ersten Erfahrungen mit der cyberdemocracy weltweit ausgewertet. Die positiven Entwicklungen, die wir für die Demokratie und die new governance des Staates anführen, sieht Pippa Norris mit gedämpftem Optimismus: „The restructered opportunities for information and communication available via digital politics will potentially have positive consequences for civic society, altering the balance of relevant resources and slightly leveling the playing field". Die meisten Vorteile sieht sie für kleine Parteien und Gruppierungen.

„Reducing the costs of information and communication minimizes some, although not all, of the significant barriers to effective political participation at individual-level; it becomes easier for ordinary citizens to learn about public affairs, if they are so inclined, and to express their views and to mobilize." 14

Weltweit wird dieser Prozeß vor allem für die sich entwickelnden Demokratien wie Taiwan, Brasilien und Südafrika von Bedeutung werden, ,if e-governance improves

${ }^{14}$ Norris 2000 . 
transparency and openness in the policymaking process." Doch weist sie auf eine mögliche, begleitende Entwicklung hin:

„But whether the Internet can ever encourage the less engaged to take advantage of these opportunities at mass level remains doubtful, because as the medium of choice par excellence, it becomes even easier for people to tune out from public life." 15

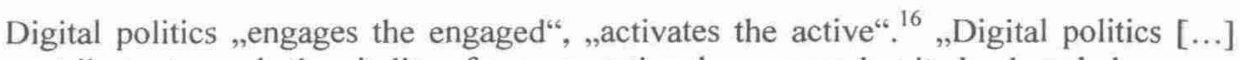
contributes towards the vitality of representative democracy, but it also largely bypasses the disengaged. "17 Der "digital divide" - so der Titel ihres Buches - wird nicht aufzuheben sein: "There will be a growing, democratic divide" in civic involvement. Far from mobilizing the general public, the Internet may thereby function to increase divisions between the actives and apathetics within societies. "18

,Digital divide' - die Teilung der Gesellschaft in Netzaktive und Netzinaktive - ist kein Argument gegen die Extension von eDemocracy und eGovernment, da es sich um eine Ausweitung von politischen Information-, Kommunikations- und Interaktionsmöglichkeiten handelt, die zu den anderen hinzutreten. Digital politics ist ein Aktivierungspotential, kein Ersatz der bisherigen politischen Formen. Aber indem sie den Politikproze $ß$ transparenter macht, mögliche Alternativen sichtbarer und die Zurechenbarkeit von Politiken verbessert, eröffnet die digital politics neue Informationsräume. „People can thereby cast informed ballots that accurately match their political preferences." 19

Der Spielraum der Bildung und Artikulation politischer Präferenzen wird größer, auch weniger institutionalisiert. Hier wachsen Befürchtungen der Destabilisation der repräsentativen Demokratie. ${ }^{20}$ Doch solche Befürchtungen können lediglich die Erwartungen neu justieren, aber nicht die Entwicklung stillstellen. Natürlich sind die Bürger nicht plötzlich bereit, das Internet zu nutzen in politischen Angelegenheiten. Vor allem fehlen noch die interaktiven Angebote. Institutionalisierte politische Konventionen ändern sich erst über neue e-governance-Angebote. In Deutschland haben wir das neue Spielfeld fast noch gar nicht eröffnet. Die Portale der Politik sind erst noch bessere Anzeigentafeln. Lediglich erste Formulare sind auf den www-Seiten abrufbar; die digitale Verwaltung beginnt ihre ersten, vorsichtigen Schritte. Wir stehen noch vor jedwelchen Erfahrungen.

Noch sind die Kommunen nicht bereit, ihre laufenden Politikprozesse auf die Portale zu legen, ihre Budgets, ihre Projekte, ihre Prioritäten, ihre Fehler, ihre Verantwortlichkeiten. Digital politics hat politikrestrukturierende Potentiale, die in Pippa Norris' groBer Studie nicht entfaltet werden. Es geht nicht nur um Transparenz und verbesserte Information, sondern um Kommunikation. Kommunikation heißt hier: ständiger Aus-

15 Ebd., S. 20.

16 Ebd., S. $18 \mathrm{f}$.

17 Ebd., S. 19.

18 Ebd., Kap. 11, 9.

19 Ebd., Kap. 6, 10.

20 Bimber sieht im amerikanischen politischen System einen Prozeß der Bewegung von interessenbasierten Gruppenpolitiken hin zu eher fluiden, themenbasierten (issue-based) Gruppenpolitiken mit geringerer institutioneller Verbindlichkeit (Bimber 1998). 
tausch von Einschätzungen - der Politik an ihre Bürger, der Bürger an ihre Politiker und ihre Verwaltung. Die laufenden Politikprozesse können als Prioritätenlisten veröffentlicht werden, mit Abstimmungen zur Änderung und Anpassung der Prioritäten. Die kommunalen Budgets können offengelegt werden.

Wenn ein neues Viertel geplant wird, können die Bewohner einer Kommune mitplanen. $\mathrm{Ob}$ eher Kindergärten geschlossen werden sollen oder Stadtbüchereien, ist unmittelbar von den Bürgern zu erfragen. Die Rückkopplung zu den Bürgern ist entscheidend. Natürlich bleibt die Politik initiativ - ob aus eigener Kompetenz oder angeregt durch die Bürger. Es geht nicht um den Ersatz von Politik durch direkte Demokratie, sondern um die Ausweitung der Politikmöglichkeiten (Wiederkehr der Politik in die Politik). Es geht bei der digital politics um neue Anreizsysteme, die Bürgerpräferenzen genauer in Politik zu übersetzen. Die Politik hat die Chance, Vertrauenspotentiale aufzubauen, die sie in den eingefahrenen Bahnen nicht mehr hat. Die Politik kann hier in soziales Kapital investieren.

Pippa Norris' Analyse, ,digital politics functions to reduce the costs for the actives becoming more active", ist realistisch bezüglich einer Annahme der Demokratisierung der Demokratie. Wir vertreten aber keinen Partizipationsidealismus, sondern die These, die Politik in die Politik zurückbringen. In Norris' Konzeption haben wir es mit einer neuen Variante des ,politischen Unternehmertums' ${ }^{6}$ zu tun. Die Aktiven erschließen sich neue Handlungsmöglichkeiten. Doch ist diese Sichtweise einseitig, weil ohne die durch die digital politics erlangten neuen Anreizstrukturen formuliert. In den Netzwerken entstehen neue Akteure: neue Gruppierungen, neue Koalitionen, neue Konstellationen, die nicht durch die Form traditioneller Interessenorganisationen prädeterminiert sind (seien es Parteien, Verbände etc.).

Nicht nur werden die Aktiven aktiver, sondern der Raum der Aktivierung weitet sich aus. Non-governmental-organizations (NGOs) - und andere, bis jetzt noch unbekannte politische Konfigurationen - können völlig neuartige Netzwerke mobilisieren. ${ }^{21}$ Yoram Barzel spricht von ,third party enforcers', die die Politik durch Drohungen der Reputationsbeschädigung steuern. ${ }^{22}$ Hier läßt sich das Internet als neue Öffentlichkeit einfügen, die die alten Öffentlichkeiten beileibe nicht ersetzt, sondern ergänzt und ausweitet. Die neuen politischen Akteure, die im Internetzusammenhang entstehen oder ihre Koalitionspotentiale neu eruieren, arbeiten wesentlich über das Medium der Öffentlichkeit, nicht über bi- oder multilaterale Absprachen. Sie arbeiten als das Gedächtnis der Politik, erinnern an Versprechungen, mobilisieren Erwartungen, nutzen die Transparenz der neuen Öffentlichkeit, um auf Präferenzen zu bestehen, die längst zu bedienen zugestanden, aber nicht eingehalten wurde.

Sie drohen, die Reputation zu schädigen, d.h. die Wiederwahl der Regierung. Greenpeace z.B. arbeitet als eine solche professionelle politische Agentur, als ,third partyenforcer'. Die ,dritte Partei', die hier ins Spiel kommt, ist diejenige, welche die Verträge zwischen Bürgern und Regierungen, die qua Wahlakt abgeschlossen werden, supervisioniert und, qua Kommunikationsmacht, zusätzliche Verträge einfordert.

\footnotetext{
$21 \mathrm{Vgl}$. Keck/Sikking 1998.

22 Vgl. Barzel 1998, sowie Wieland 1999.
} 
Das Internet gebiert politisch eine Mannigfaltigkeit von möglichen ,third parties ${ }^{*}$, deren Partizipationseinklinkung über das Medium Öffentlichkeit läuft. Das Internet ist darin eine Ausweitung der Öffentlichkeit, über die der gewöhnlichen Medien hinaus. ,Third-party-enforcers' betreiben wesentlich, issue-management ${ }^{*}$ : sie setzen Themen, fokussieren Probleme, stellen Fragen, die unbeantwortet sind. Ihr Erfolg besteht darin, einer Politik ein Thema nahezulegen bzw. aufzudrängen. So greifen sie in die Politik ein, intervenieren, ohne zu intervenieren.

,Third-party-enforcers" sind unspezifische oder atypische politische Akteure. Sie können als politische Agenturen bekannt sein, aber auch aktuell sich neu formieren: als politische Kampagnen z.B., als unabhängige Wähler etc. Wir können nur die Struktur der Netzwerkpolitikpotentiale beschreiben: Erst wenn der Staat das eGovernment ausgebaut hat, wird sich zeigen, welche neuen Interaktionsformen entstehen. Wenn die kommunalen oder anderen politischen Portale zur Kommunikation auffordern, bilden sich die Gruppen, die der Aufforderung folgen, unabhängig und parallel zu den ,üblichen Verdächtigen': den Parteien, Verbänden etc. eGovernment und die dazugehörige eDemocracy-Komponente sind Angebote, die sich ihre Nachfrage bilden werden. Wenn die interaktiven Modelle konkretisiert sind, bilden sich die Formen, die sie bedienen. Die neuen Handlungsmöglichkeiten müssen entdeckt werden. Das Spiel wird eröffnet werden, aber ist noch unexperimentiert. Bei den NGOs - extensiven Nutzern des Internets - liegen erhebliche Erfahrungen vor. Wir haben hier längst eine ePolicy-Extension, deren Agilität und Thematisierungseffektivität die Potentiale einer eDemocracy bereits jetzt schon skizziert.

Das Spiel der neuen Kooperationsformen zwischen Bürgern und Politik ist wesentlich eine Form des Wissensaustausches. Unvorsichtigerweise verwenden wir gerne das Wort Wissensgesellschaft, ohne die Konklusionen und Implikationen zu kennen. Die verbesserten Informationen und die möglichen Kommunikationen sind nur die Folien eines Prozesses, der dem zugrundeliegt. Wenn wir, wie wir bereits entwickelt haben, Steuerungsdefizite des Staates konstatieren, wenn wir an die Grenze der Interventionskompetenz des Staates gelangen, weil er nicht weiß, welche Regulierungen und Eingriffe welche Resonanz und Effekte erzeugen, ist er darauf angewiesen, seine Adressaten zu fragen, was sie brauchen, mit dem Vorbehalt, unter der Budgetrestriktion gleichzeitig fragen zu müssen, was sie bevorzugen, und was sie hintanzustellen bereit sind. Digital politics bzw. eGovernment ist dann die neue Kooperation des Staates mit den Bürgern, sie unmittelbar, in jedem Projekt, in jeder Politik, zu fragen, was sie bevorzugen angesichts der Tatsache, nur begrenzte Politiken realisieren zu können.

Natürlich muß die Regierung weiterhin die Entscheidungen fällen. Das kann von keiner direkten oder, strong democracy“ übernommen werden. Aber die Vorbereitung ändert sich. Nicht mehr nur die Wahlen entscheiden, was die Regierung tut, sondern sie fragt im laufenden Politikprozeß nach, was konkret jeweils zu verwirklichen angemessen ist. Digital politics ist Politikprozeßkommunikation, nicht nur Wahlkampfkommunikation. 


\section{3. eGovernment}

eGovernment auf eine Variante des, lean state` zu reduzieren, erschließt seine Möglichkeiten und Potentiale nur unzulänglich. Es geht nicht nur um eine - IT-gestützte Verschlankung des Staates, wesentlich seiner Verwaltung, sondern um die Reform des Staatstypus (und damit natürlich auch um Verwaltungsreform) und neue Beziehungen zu den Bürgern, die durch die eDemocracy entfaltet werden. Es wäre undurchdacht, lediglich die Kosten des Staates zu senken, und die gleichen Leistungen wie bisher zu erwarten. Was der Staat leisten kann und soll, und wer es leisten kann und soll, steht zur Diskussion. Bei James G. March und Johan P. Olsen finden wir eine Unterscheidung nach vier Staatstypen, anhand derer sich das eGovernment neu einreihen läßt:

1. the sovereign state,

2. the institutional state,

3. the supermarket state model,

4. the corporate bargaining state. ${ }^{23}$

March/Olsen definieren mit ihren vier Staatstypen vier Organisationsmuster, die das Verhältnis von Politik, Verwaltung und Bürger jeweils anders konstellieren.

$\mathrm{Zu}$ 1.: The sovereign state:

„Die Rolle des Staates besteht hier darin, die Gesellschaft nach politischen Präferenzen und Konzepten einer gerechten Gesellschaftsordnung zu gestalten. Gewonnene Wahlen geben der politischen Führung Autorität und legitime Gestaltungsmacht, als Architekt der Gesellschaftsordnung zu fungieren. Die Verwaltungsorganisation hat daher instrumentalen Charakter. Die staatliche Bürokratie fungiert als neutrales Instrument zur Durchsetzung politischer Ziele. Das Standard-Organisationsmodell ist die Ministerialverwaltung, eingebettet in , a hierachy of influence, responsibility and control'، "24

$\mathrm{Zu}$ 2.: The institutional state:

„Die primäre Aufgabe des Staates besteht in diesem Modell darin, die bestehenden politischen und gesellschaftlichen Strukturen zu gewährleisten und die Autonomie der verschiedenen institutionell umhegten gesellschaftlichen Bereiche zu respektieren. Im Gegensatz zum vorherigen Staatstyp ist der Charakter der Verwaltungsorganisation nicht instrumentell zu verstehen. Institutionen stellen sich dar als Träger von Kulturen, Werten und Identitäten. Organisationen werden nicht planvoll gestaltet oder , designed ${ }^{*}$, sie entwickeln sich langsam in einem gewissermaßen natürlichen historischen Prozeß. Institutionenveränderungen sind weniger das Ergebnis geplanter Organisationsentscheidungen, sondern stellen sich als schrittweise, allmähliche Anpassung an Veränderungen der Organisationsumwelt dar. In der Regel vollziehen gezielte Organisationsveränderungen nur bereits tatsächlich erfolgte Veränderungen nach. Sie sind - so March und Olsen - symbolic and rhetorical events that educate the public and change the climate of opinion'.".25

${ }^{23}$ March/Olsen 1989.

${ }^{24}$ Schuppert 1998, S. 30.

${ }^{25}$ Ebd., S. $30 \mathrm{f}$. 


\section{Zu 3.: The supermarket state model:}

„Im sogenannten Supermarkt-Modell besteht die Rolle des Staates darin, auf dem Markt als einer von mehreren Anbietern von Gütern und Dienstleistungen aufzutreten. Die die Organisationsentscheidungen steuernden zentralen Werte heißen Überlebensfähigkeit, Flexibilität, Wirtschaflichkeit und Effizienz. Das Zauberwort heißt Wettbewerb. Das Standard-Organisationsmodell ist das auf einem Wettbewerbsmarkt operierende öffentliche Unternehmen. Verwaltungseinheiten sind nur ein Typ unter anderen vom am Markt handelnden Akteuren. Die Verwaltungskultur verändert sich , from an administrative culture to a service culture ${ }^{6.626}$

$\mathrm{Zu} 4 .:$ The corporate bargaining state:

„Im korporatistischen Verhandlungsstaat vermitteln gewonnene Wahlen nicht wie im Modell Nr. 1 ein umfassendes Mandat zur Gesellschaftsgestaltung, sondern ,nur` einen Platz am Verhandlungstisch, und die formale Hierarchie ist nur ein Kontrollinstrument unter anderen. Die organisierten Interessen haben direkten Einfluß auf die Formulierungen und die Umsetzung politischer Programme. Die staatliche Bürokratie ist nicht mehr unparteiliches Instrument, die öffentlichen Bediensteten werden zu ,key players' im politischen Prozeß als Repräsentanten von Institutionen, Werten und Klienten. Ihr Rollenverständnis ist nicht das des unparteilichen Richters, sondern sie agieren als Schlichter, Verhandlungsführer und Konfliktmittler. [...] Anders ausgedrückt ist der Staat zunehmend Moderator politischer Entscheidungsprozesse, ist er - um es auf den Begriff zu bringen - ,état catalysateur', so muß dies auch auf seine Verwaltung, ihre Handlungsformen, ihre Steuerungsinstrumente, ihren Handlungsstil durchschlagen: dem ,état catalysateur' entspräche eine ,administration catalysatrice', dem vermittelnden Staat eine vermittelnde Verwaltung. “27

Der aktuelle Staat ist - nach diesem Kriterienraster von March/Olsen - ein Hybrid, mit etlichen Verschiebungen und Übergangsbewegungen. Natürlich haben wir im aktuellen Staat noch viele Elemente des, sovereign state' (I.), auch im Selbstverständnis von Politik und Verwaltung. Der , sovereign state ist identisch mit dem klassischen deutschen Staatsverständnis, inklusive seiner politisch neutralen Beamtenschaft.

Doch arbeitet der Staat faktisch längst anders, auf allen - kommunalen, Länder- wie Bundesebenen. Viele Regierungs- und Verwaltungseinheiten arbeiten z.T. längst nach dem Supermarkt-Modell (III.), mit outgesourcten Verwaltungseinheiten, die als öffentliche Unternehmen marktfähig wurden.

(I.) und (III.) bilden ein Spektrum von entgegengesetzten Staatstypen, die in ihrer Gegensätzlichkeit dennoch Mischungen in ein und derselben Staatseinheit bilden. Der Staat ist längst in Bewegung, auch in den Dimensionen (II.) und (IV.), die das Mischungsverhältnis anreichern. Der aktuelle Staat der BRD ist, nach dem Schema von March/Olsen, eine heterarchisch gemischte Staatstypenkomplexion.

Der ,institutional state' (II.) gibt Regeln, innerhalb derer die Akteure (Politik, Verwaltung und Bürger) frei agieren. Auch dieses Moment kommt in der hybriden Organisation des aktuellen Staates vor, wenn auch nicht dominant. Am Stärksten haben wir aber innerhalb von IV. eine Entwicklungsänderung: der ,corporative bargaining state

\footnotetext{
${ }^{26}$ Ebd., S. 31 f.

27 Ebd., S. 33 f. Zum Topos des ,état catalysateur‘ vgl. Keane 1988, S. 1-30.
} 
wandelt sich vom korporatistischen Arrangement (kooperativer Staat ${ }^{28}$ ) (IV.a) über die Verhandlungsdemokratie (IV.b)zum moderierenden Staat (IV.c). ${ }^{29}$

Der Veränderung des Staatstypus IVa - IVc laufen organisatorisch adaptierte Verwaltungsformen parallel:

(IV.a) Kooperativer Staat - kooperatives Verwaltungshandeln

(IV.b) Verhandelnder Staat - aushandelnde Verwaltung

(IV.c) Moderierender Staat - konfliktvermittelnde Verwaltung

In der letzten Zustandsform des Staatstyps IV. treten

„Staat und Verwaltung [...] nicht primär als Verhandlungspartner auf den Plan, die in zweioder mehrpoligen Verhandlungssystemen zu den Verhandlungspartnern in tauschförmige Beziehung treten, sondern Staat und Verwaltung agieren als Organisator und Moderator von Verhandlungsprozessen. ${ }^{\text {“30 }}$

Gunnar Folke Schuppert reformuliert hier die Ergebnisse der Steuerungsdiskussion in der Soziologie ${ }^{31}$ und Ökonomi ${ }^{32}$ als Extension des March/Olsen-Staatstypus' IV. Dietrich Fürst hebt heraus,

„daß der Staat zunehmend weniger autonom steuern kann, sondern zunehmend in die Rolle des Moderators von laufenden Prozessen gedrängt wird, wobei seine wichtigste Aufgabe darin besteht, die Handlungsprämissen dieser Prozesse zu beeinflussen, insbesondere die Spielregeln für gesellschaftliche Problembearbeitungsprozesse vorzulegen. ${ }^{\text {.33 }}$

Bruchlos geht diese Konklusion über in die Definition der neuen Rolle des Staates bei Robert Sudgen, die sich in der institutionenökonomischen Theorie wiederholt: „The primary role of government is not to maximize the social good, but rather to maintain a framework of rules within which individuals are left free to pursue their own ends. ${ }^{\text {“34 }}$ Letzthin haben wir Sudgens Proposition nur etwas subtiler ausbuchstabiert.

Josef Wieland bringt einen neuen Aspekt, der scheinbar nur die internationale Dimension betrifft:

„Die Organisationen des Politik- und Rechtssystems [...] können sich entweder nicht vom Territorialprinzip lösen oder nur mit erheblichen Zeitbudgets und anderen Transaktionskosten. Dazu zählen vor allem der Zugriff auf relevante Informationen und Wissen als Voraussetzung der effizienten Gestaltung globaler Ordnungspolitik, das immer häufiger nicht im öffentlichen, sondern im privaten Sektor der Gesellschaft zu finden ist. Entweder gelangt die Politik nicht in den Besitz der notwendigen Expertise oder aber nicht schnell genug. Beides läuft der Temporalisierung des Wirtschaftslebens und der Ökonomisierung der Zeit als dynamische Ressource diametral entgegen. Ökonomisch gesehen steigen durch die sich daran anschließende ineffiziente Anpassungsfähigkeit der weltweiten staatlichen institutionellen Rahmenordnungen die

${ }^{28}$ Ritter 1979; Schuppert 1998, S. 36 ff.

${ }^{29}$ Schuppert 1998, S. 36 ff.

${ }^{30}$ Ebd., S. 41.

31 Vgl. z.B. Willke 1992, 1997

32 Vgl. z.B. Wegner 1996, Noll/Ebert 1998.

${ }^{33}$ Fürst 1987, S. 27.

34 Sudgen 1993, S. 1948. 
Transaktionskosten (Anbahnung, Durchführung, Überwachung und Erzwingung von Vertràgen) der globalen Tauschhandlungen. Privatwirtschaftliche Arrangements oder die Delegierung von Aufgaben an andere gesellschaftliche Organisationen und Unternehmen innerhalb eines politischen Netzes sind dann transaktionskostensenkende, funktionale Äquivalente. ${ }^{35}$

Was Wieland für den staatsfreien internationalen Raum beschreibt, enthält viele Momente auch der nationalen Verschiebungen zwischen Staat und Gesellschaft. Ohne weitreichende Reorganisation von Politik und Verwaltung erhöhen sich auch national die Transaktionskosten der Regulation und Ordnung, im zunehmender Spannung zur Temporalisierung der Wirtschaft. Die new economy, aber auch die Formen der Virtualisierung der Geschäftsbeziehungen in der old economy, erfordern neue Rechtsgrundlagen, neue Ordnungsprinzipien, neue Vertragsräume, neue Infrastrukturinvestitionen (vor allem der Bildung), die der langsame Staat nicht liefern kann oder so verzögert, daß er als entwicklungshemmend auffällt. Auch hier werden sich privatwirtschaftliche Arrangements entfalten, die funktional die Aufgaben übernehmen, die man vom Staat zur Zeit nicht mehr erwartet: eigene Commitments, eigene Normen, eigene quasiRechtsinstitutionen, eigene Finanzierungsmodelle etc.

Das Internet - das Wieland gar nicht anspricht - stellt sich als soziales Medium einer schnellen Arrangierung dar. Es fungiert als nicht-klassischer Diskursraum der Rekonstellierung von Absprachen und Arrangements - ein Raum der privaten Politiken dort, wo die staatliche Form der Politik nicht, oder noch nicht, präsent ist.

Das Internet wird zum großen Forum: für Sinn und Unsinn. Aber es bietet eine Arrangierungsarena von bisher unbekannter Novität, Reichweite und Ankopplungsmöglichkeit, die deshalb transformativ wirkt, weil sie die Gesellschaft aus den eingefahrenen, konventionellen Netzwerken in den Möglichkeitsraum unerwarteter, neuer Netzwerkarrangements bringt, die nicht von vornherein durch die Filter der alten Eliten besetzt sind. Es wäre unbedacht, auszuschließen, daß sich hier neue (Netzwerk-)Eliten bilden. Die eDemocracy ist keine Verkörperung reiner Demokratie, sondern eher nur eine Änderungsagentur: sie distributiert die political entrepreneurs neu, bringt neue political agents ins Spiel, verwirbelt die klassischen Strukturen.

Sie bringt andere Akteure ins Spiel, ist eine Plattform, die den Zugang zur Politik durch Rekonstellierung der Netzwerke erweitert, ohne daß man sich der Illusion hingeben bräuchte, alle Bürger träten in den politischen Diskurs ein. Die Extension des politischen Raumes, wenn wir eDemocracy erst einmal so einführen, ist kein Effekt, der durch Verallgemeinerung der politischen Teilhabe entsteht, sondern vornehmlich, zumindest ersteinmal, durch Änderung der Netzwerke, durch Substitution. Neue Spieler treten in die Arena, die sich über die gewöhnlichen Zugangskarrieren - politische Parteien - niemals eingefunden hätten.

Pippa Norris wurde oben deshalb so ausführlich zitiert, weil ihre Ergebnisse einer international vergleichenden Studie über den digital divide auf genau diese Konsequenz hinauslaufen: die Aktiven werden zusätzlich aktiviert, ${ }^{36}$ die Inaktiven verstärkt inakti-

35 Wieland 1999, S. 15.

36 Man schlage nur die Zeitschrift ,Greenpeace Magazin' auf, z.B. Heft 2/2001, S. 20 f., zeigt eine Weltkarte von Internetadressen, die unter dem Titel „www.weltweiter.widerstand“ das Netzwerk 
viert. eDemocracy hat wenig mit Demokratieidealismus zu tun, sondern bedient ein anderes Thema: Macht- und Elitenverschiebung.

Doch wäre es unvollständig, es so konventionell einzuordnen. Indem neue politische Eliten sich konfigurieren können, mobilisieren sie auch neue Klientel. Oder noch anders gesagt: um reüssieren zu können, müssen sie mit neuem Klientel antreten. Das kann in der Mobilisierung neuer, bisher inaktiver Bürger funktionieren, oder aber in der Rekonstellierung bisher bereits aktiver: durch Abzug von Wählern von Partein auf neue Themengruppierungen hin. Die Netzwerk-Politik kann mit einer Drohung arbeiten, die bereits dann wirksam wird, wenn sie noch gar nicht mit ,eigenen Truppen auftritt. Die Drohung lautet: ,Ich ziehe Dir Truppen ab.' In einer Welt der Mediendemokratie sind Thematisierungsvorstöße, mit gutem Medienmanagement, für die etablierten Parteien riskant. Sie können ,Ränder gefährden'. Netzwerkpolitik braucht nicht als Partei aufzutreten, sondern es reicht, wenn sie Themenherrschaft gewinnt, um Wechselwähler oder lose Gekoppelte ,abzuwerben'. Netzwerkpolitik bringt Turbulenzen ins Spiel, die nicht durch die gewöhnlichen Muster und Politikspiele abgearbeitet werden können.

\section{New state}

Doch interessieren uns diese staats- und politikwissenschaftlichen Kautelen nur insoweit, wie sie die eGovernment-Dimension nicht als lediglich informationstechnologische, von außen kommende Innovation kennzeichnen, sondern als nahtlose Einloggung in eine schon länger laufende, vom Internet unabhängige Entwicklung und Änderung des Staatstypus.

Denn wenn der Staat vornehmlich als Moderator auftritt (und seine Verwaltung als konfliktvermittelnde Instanz), setzt dies voraus, daß die Gesellschaft und ihre Wirtschaft sich auf ein $\mathrm{Ma} ß$ an Selbstorganisation einstellen, das nicht mehr erwartet, mit einem intervenierenden Staat zu rechnen. Der Staat (und seine Verwaltung) treten stärker als kommunikativer Staat in Erscheinung (und zwar in allen Dimensionen von IV.) ${ }^{37}$ ohne die Regulierungsfunktion aufzugeben. ${ }^{38}$ Der Staat nähert sich nicht nur als, new public management ${ }^{39}$ - Managementkonzepten, wie sie in der Privatwirtschaft längst praktiziert werden, sondern trifft als moderativer Staat auf Netzwerke ${ }^{40}$, die nicht mehr den klassischen Korporationen entsprechen: ${ }^{41}$ auf Unternehmensnetzwerke in

der Globalisierungsgegner notiert. Die dort verzeichneten 26 websides sind z.T. nur Netzwerknotenpunkte für Subnetzwerke. Die NGOs haben die Netzwerkpolicy längst erobert.

${ }^{37}$ Schuppert 1998, S. 44f f., was vor allem Folgen hat für die Änderungen von Verwaltungsverfahren.

${ }^{38}$ Ebd., S. 46 ff.

39 Budäus 1998b.

$40 \mathrm{Vgl}$. Schupperts abschließende Konklusion der „Entgrenzung des Verwaltungssystems durch symbiotische Organisationsstrukturen und Netzwerke" in Schuppert 1998, S. 58 f., mit Bezug auf Kenis/Schneider 1996.

41 Kritisch dazu Priddat 2001. 
verschiedensten Ausprägungen, auf Dritt-Sektor-Netzwerke - und auf die neuen Netzwerkpotentiale des Internet.

Fritz Scharpf hat den ,verhandelnden Staat' in vier spezifische Modalitäten unterschieden, die diese Konklusion genauer bestimmen lassen:

(a) Selbstregulierung,

(b) Bilaterale Verhandlungen,

(c) Trilaterale Konstellationen,

(d) Pluralistische Politiknetzwerke. ${ }^{42}$

Scharpf unterscheidet vier Verhandlungssystemmilieus mit von a - d zunehmenden Kooperationsniveaus: (a) bezeichnet Verhandlungen der Bürger untereinander, (b) die gewöhnlichen Einzelverhandlungen organisierter Interessengruppen, die auf direkter Interessenvermittlung bzw. auf Lobbyarbeit beruhen, (c) die tri- oder multipartistischen korporatistischen Arrangements und (d) die Politiknetzwerke, die sich nur teilorganisiert und temporär ausbilden, oft auch als laterale Substruktur quer zu allen Interessenorganisationen, als informelle Kontaktnetze.

Auch hier liegt der Übergang zum Internet auf der Hand - nicht nur als technische Basis, sondern als Potentialstruktur der jederzeit möglichen neuen Netzwerkbildung, unabhängig von den manifesten organisierten Interessen. Das Internet wird - wie die korrekte Übersetzung lautet - zum ,Zwischennetz' in den vorhandenen Politiknetzwerken und organisierten Interessenvertretungen. Es erlaubt, die Netzwerke in den Netzwerken ständig neu zu konfigurieren, in neue Kommunikations- und Koalitionszusammenhänge zu gehen.

Im eDemocracy/eGovernment-Kontext bilden die pluralistischen Politiknetzwerke eine neue politische Plattform, auf der potentie!! neue Verhandlungssysteme entstehen (und wieder vergehen) können. Die Politik kann Themen anbieten, aber die Bürger - als policy-net-communities - bieten ebenfalls Themen an, die der Staat behandeln sollte. Hier entscheidet sich, ob die Bürger-net-communities mit dem Staat zusammenarbeiten oder ob sie das, was sie mit dem Staat verhandeln wollten, selber organisieren.

Es wird deutlich, daß in der Netzwerkdimension ein neues Politikverständnis aktiver Bürgerschaft greift. Scharpfs Liste der zunehmend kooperationsintensiven Verhandlungssysteme bildet im Grunde einen Kreis - Selbstorganisation und Politiknetzwerke liegen nahe beieinander, und der Staat liegt nicht mehr notwendigerweise im Mittelpunkt der Politik.

Wir bewegen uns in die Dimension einer Politik ohne Zentrum. Es geht nicht um Staatsabschaffung, sondern darum, daß der Staat ein Akteur unter anderen in der Politik wird, der nicht notwendig der zentrale Adressat von Politikanforderungen bleibt. Netzwerkkommunikation ist keine zentristische Adressierung, die immer nur den Staat meint, sondern eine, die Kooperationsmodi finden kann, die staatsunabhängige Lösungen generieren.

Der Staat ist zwar längst dabei, seinen Typ zu wechseln, weil er seine Steuerungsund Regulationsfunktion nicht mehr ohne Kooperation und Kommunikation mit den

42 Scharpf 1993, S. 36 f. 
Adressaten seiner Politik gestalten kann, ohne Gefahr zu laufen, in seinen Politikintentionen unterlaufen oder umgangen $\mathrm{zu}$ werden. Aber erst über die eGovernmentKonzeption, die nur auf der Internetbasis entwickelt werden konnte, kann die NetzwerkKooperation ihre Fokussierung auf organisierte Interessenverbände aufheben.

\begin{abstract}
„Potentially these changes can have profound consequences for altering the balance of resources and powers between insurgent challengers and established organizations within the political system. Hierarchical communication channels, typical in bureaucratic organizations like government departments and international agencies, become less effective and slower mechanisms of information transmission than horizontal networks shared by informal coalitions of alternative social movements." 43
\end{abstract}

Erst das Inter-Netzwerk ermöglicht, nicht-organisierte Interessen in die Kooperationen, Verhandlungen und Moderationen hineinzunehmen, d.h. eine Plattform der Staat/Bürger-Relation anzubieten, die jede Kommunikation - unabhängig von organisierten Formen der Politik - aufnehmen und in den kommunikativ-regulativen Prozeß einspeisen kann.

\title{
5. eDemocracy und eGovernment als, virtual political system
}

An diesem Punkt gehen eGovernment und eDemocracy eine Liasion ein. Wenn der Staat die Bürger stärker in die Entscheidungen hineinnehmen will, beginnt er das nicht, weil er neue Demokratieprinzipien einführen möchte, sondern um seine Steuerungskompetenz zu erhöhen. Es geht beim eGovernment nur marginal um elektronische Wahlverfahren (wie sie nach dem Desaster der US-amerikanischen Präsidentschaftswahlen - völlig angemessen für dieses Problem - in Ersatz der manuellen und mechanischen Verfahren erörtert werden), ${ }^{44}$ sondern um die internetbasierte Hineinziehung der Bürger in Planungs- und, vor allem, kommunale Entscheidungsprozesse. Erst die Universalkommunikationsplattform Internet ermöglicht es, ohne spezifische Kostenerhöhungen Befragungen und Wahlen an die Bürger heranzutragen, in kommunikative Interaktion zu gehen, so daß das Wahl-, Beratungs- , Planungs- und Entscheidungsprozedere in neuen Formen ineinandergreift.

"The main democratic potential of digital information and communication technologies lies in strengthening organizational linkages and networking capacities in civic society, tipping the balance of relevant political resources form money, members and bureaucratic organizations to know-how and technical skills, providing more open and pluralistic forms of competition for insurgents among parties, interest groups, alternative social movements and the independent media, and expanding the resources of information released by government departments, official bodies, and international agencies. This process alters the opportunities for the institutions of civic society, particularly insurgents, to network horizontally with each other, to challenge the authority of established institutions, and to reinforce their linkages with activists. ${ }^{45}$

${ }^{43}$ Norris 2000, S. 16.

${ }^{44}$ Ebd.

${ }^{45}$ Ebd., S. 17. 
Dadurch ist eine Flexibilität und Unkonventionalität der eingefahrenen Bürger/StaatBeziehungen möglich - unabhängig von klassischen Interaktionskanälen, offen für neue Akteure -, die in den klassischen Verbänden und Parteien nicht vorkommt. Die klassischen Koalitionen werden auflösbar, das politische Spiel neu durchmischt, mit neuen Konfigurationen und , unwahrscheinlichen Konstellationen‘. ${ }^{46}$

eGovernment, und eDemocracy, sind - neben allen Kostenreduktionsgesichtspunkten der , lean state“-Debatte - nicht nur Verstärker der new governance, d.h. der Änderung des Staatstyps vom kooperativen zum moderativ-kommunikativen Staat, sondern die Materiatur der Selbstorganisationspotentiale, die den Staat in die Rolle des Moderators überhaupt erst sich zurückziehen läßt. Denn in dem Maße, in dem die Bürger stärker in Planungs- und Entscheidungsprozesse hineingenommen werden, tritt der Staat von seiner regulativen Rolle zurück und übernimmt eine moderative Rolle.

Erst über das politisch offerierte eGovernment und seine Internetpotentiale können sich neue Konstellationen und Koalitionen bilden, die vorher keine Projekt- und Interaktionsfläche hatten. eGovernment wird zum Politikangebot, an dem die gesellschaftlichen und wirtschaftlichen Akteure sich neu konfigurieren lernen. Der Staat schafft sich seine Moderationspopulation, indem der die Bürger einlädt, über das eGovernment/Internet

1. ihre Politikartikulationen präziser zu tätigen und

2. untereinander zu verhandeln, welche Politik sie selber machen, anstatt sie dem Staat zu überlassen.

Erst dann lassen sich neue Kooperationsmodalitäten ersinnen, bis hin zu neuen Formen der eDemocracy, die die korporatistischen Politikmodelle weiterführen, aber in veränderter, völlig neuer Form: als kooperative Netzwerkkonstellationen, die themenzentriert und lokal Problemlösungen arrangieren. Es geht dann um Problemlösungen der aktuellen Netzwerkpopulation, über Parteien und Verbändeinteressen hinaus. ${ }^{47}$

Der sich derzeit abzeichnende Trend zu einer eDemocracy und einem eGovernment ist keine Mode, die wieder verschwindet. ${ }^{48}$ Sie hat das Potential zur radikalen Veränderung, zu einer grundlegenden Reorganisation für das Verhältnis Bürger/Politik und Bürger/Verwaltung, mit Konsequenzen für das Verhältnis Politik/Verwaltung. Ihre neue Qualität ist ihre Netzwerkstruktur, die keinen Hybrid zwischen Markt und Staat oder Markt und Organisation bildet, sondern eine Koordinationsform eigener Qualität. ${ }^{49}$ Damit wird weiterhin um die Paradoxie gerungen, daß die Bürger einerseits Souverän der Politik sein sollen, andererseits als Interventionsempfänger unter den Verwaltungen leiden müssen. Wenn die Verwaltung so organisiert ist, daß sie als Bürgerpartner neu legitimiert wird,

${ }^{46}$ Der Finanzwissenschaftler Gunther Engelhardt entwickelt hierzu eine Konzeption , symbiotischer Arrangements' (Engelhardt 1995).

${ }^{47}$ Der politische Korporatismus legitimierte sich dadurch, daß die Verbände nicht nur die Interessen ihrer Mitglieder, sondern auch Nichtorganisierter vertraten (vgl. Priddat 2001). Das wird überflüssig, wenn die eGovernment-Netzwerke alle die in die Politik holen, die thematisch interessiert sind. Die Demokratie wird direkter, unvermittelter. Und zwar ohne normative Bemühungen um Plebiszitarität.

${ }^{48}$ McDonough 2000, S. 9.

49 Powell 1990, S. 217 f.; Weiß 2000, S. 78 ff. 
kann die Politik sich das zuschreiben; zugleich aber ändert sich die Anforderung an die Politik auf umsetzungsfreundliche Projekte hin.

Es geht um ein neues Politikmodell: the virtual political system. ${ }^{50}$ Die wichtigsten Ergebnisse der Politikmodelle des ausgelaufenen 20. Jahrhunderts lauten: „the rediscovery of incentives, information problems, and complexity. "51 Die technologische und soziale Netzwerkstruktur wäre in dieser kleinen Liste zu ergänzen. Sie bildet die Plattform für das eGovernment. eGovernment ist aber keine neue Regulationsform des Staates, sondern eine netzwerkformierte Interaktionsfläche vieler möglicher neuer Beziehungen zwischen Staat, Politik und Bürgern.

David G. Post und David R. Johnson haben eine theoretische Konzeption dieses neuen governance-Prozesses vorgelegt. Das traditionelle

\begin{abstract}
„ideal of rational debate among wise elected representatives regarding the overall public good may be replaced, online at least, by a new architecture of governance that allows dispersed and complex interactions among groups of individuals taking unilateral actions and seeking more local goals and solutions. Instead of attempting to rely even upon the best of our democratic traditions to create a single set of laws imposed on the net from the top down, we may all be better off if we allow the emergence of diverse and contending rulesets, in distinct areas of the net, which pull and tug against each other (and help to recruit or drive off potential participants) - with the result that an optimal overall combination of rule arises. Rather than relying on good citizens of the global electronic polity to debate thoughtfully about a single shared vision of the common good, we might do better to look for a form of civic virtue that can reside in the very architecture of a decentralized, diverse complex system, like the net. ${ }^{\text {s2 }}$
\end{abstract}

Die vielfach angekündigte Bürger- oder Zivilgesellschaft könnte sich in einer eDemocracy so konkret als - multipel verteiltes - lokales Politikportal der Bürger dargeben. Das Internet, zunächst nur eine Technologie, wird zur Infrastruktur der eCommunities for Communities - der elektronischen Gemeinschaften von Kommunen, in der die erodierte Glaubwürdigkeit durch ein Partizipationsmoment zurückgewonnen werden könnte. 53

In unserer Demokratie wird die Produktion öffentlicher Güter mehr oder minder automatisch an den Staat delegiert; in einer Zivilgesellschaft kann der Auftrag an verschiedene Organisationen erteilt werden, z.B. auch an private Unternehmen, an die

${ }^{50}$ Norris 2000 , S. 13.

51 Eggertson 1997, S. 14.

52 Post/Johnson 1997, abstract. Das Modell beschreibt die neue Form der Politik als Politikdiversifikation, die ihre kommunikative Struktur im Internet hat, die rulesets aber lokal verteilt sich entwikkeln läßt. Post und Johnson simulieren die e-democracy nicht als IT-unterstütztes Verfahren der Verbesserung der vorhandenen Formen der Demokratie, sondern stellen die Politik ganz auf Netzbetrieb um, innerhalb dessen die Bürger in thematischen, meist lokalen net-communities evolutive Politikmuster (inklusive von Gesetzen und Verordnungen: rulesets) generieren, die - das ist Post/Johnsons neue Politversion - wechselwirkend zu generellen Politik- und Regelmustern zusammenwachsen (bottom up). Natürlich ist das eine idealtypische Konzeption, die aber die Relation Netzwerk und Selbststeuerung als komplexes Interaktionsgebilde thematisiert, allerdings über die Steuerungsgröße ,Bürgertugend" (civic virtue) konfirmiert, die als stabile innere Ordnung den Wegfall des großen Staates als äußere Ordnung kompensieren soll.

53 Vgl. Leib 1998; von Korff 1998; Jarren 1998. 
Bürger selber (in zu gründenden Organisationsformen). Es entsteht ein Wettbewerb möglicher Realisatoren der Kollektivgüter. Die scheinbar klare Unterscheidung Staat bzw. Non-Profit-Organisationen auf der einen Seite und marktwirtschaftliche Organisationen auf der anderen Seite verschiebt sich in ein neues Feld der für Industrieunternehmen bereits bekannten co-opetition: Kooperation und Kompetition werden auch in der Staatswirtschaft parallel laufen. ${ }^{54}$

Nichts spricht dagegen, die Aufgaben an die Bürger zu delegieren, wenn geeignete Organisations- und Kooperationsformen angeboten werden, die die Aufgabenerledigung stabilisieren. Ehrenamt als idealistischer Ausbeutungszusammenhang ist zunächst ein interessanter Anknüpfungsknoten, doch für stabile Kooperationsformen haben wir in diesem Bereich nur wenige viable Modelle und wenige Ressourcen. Das heißt nicht, $\mathrm{da} ß$ es nicht sinnvoll wäre, die Kooperationsressourcen zu bilden, zu fördern und zu entwickeln. Doch können wir nicht davon ausgehen, daß die staatsleistungsgewöhnten Bürger schnell und leichterhand in der Lage sind, die organisatorische Kompetenz auszubilden, die nicht zu liefern sie den Staat gerade kritisieren. Das Feld des co-opetition wird sich aber vor allem durch die neuen Steuerungsformen in einer vernetzten Zusammenarbeit von Wirtschaft und Politik bei der Erstellung von öffentlichen Gütern ergeben. Denn marktwirtschaftliche Wirtschaftsorganisationen - immer noch stabiler als Bürgerorganisationen - werden als Wettbewerber um diese Leistungen zu Kooperationspartnern des Staates. In einem durch Kosten und Gemeinwohlsvorstellungen bestimmten Aushandlungsprozeß zwischen Staat, möglicherweise bestehenden Parafiski als intermediäre Organistionen und interessierten Unternehmen wird die Wertschöpfungskette neu konfiguriert. Die Unternehmen sind auf das Wissen und die Kooperation mit dem Staat nach wie vor angewiesen, dennoch kommt das Moment einer effizienten Wettbewerbsorientierung ins Spiel. Diese Steuerungsform verlangt aufgrund der Komplexität eine hohe Kompetenz von beiden Seiten - auf der der wirtschaftlichen und der der politischen Unternehmer. In einem radikalen Verständnis des eGovernment müßten Bürger und Wirtschaftsorganisationen öffentlich einen erneuten Aushandlungsprozeß fordern können, so daß auch der Status quo argumentierbar bleibt.

eGovernment ist keine Fortführung der alten Regierungsformen mit elektronischen Mitteln, sondern eine Änderung der governance-structure der Politik, die mit den Namen ,Verhandlungssystem" und ,Netzwerk' synonym beschrieben werden können. ${ }^{55}$ Wir hatten oben bereits - in der Darlegungen der March/Olsen'schen Staatstypen - den , corporate bargaining state' mit Netzwerkstrukturen verknüpft. ${ }^{56}$

„Als spezifische Interaktionsformen erlauben Verhandlungen sowohl das Abwägen konfligierender als auch die Formulierung gemeinsamer Ziele, die durch Kommunikation - in Hirschmans Terminus: Voice - aber auch durch die Drohung mit einem Exit realisiert werden könnten. Die Möglichkeit wechselnder Koalitionen in Netzwerken macht diese gleichzeitig flexibler als Organisationen. Insofern Netzwerke daher theoretisch einerseits auf externe Anforderungen flexibel reagieren können, in ihren Reaktionen aber andererseits für externe Be-

\footnotetext{
${ }^{54} \mathrm{Vgl}$. zu einer erweiterten Diskussion des co-opetition Jansen 2000 sowie die Beiträge in Jansen/Schleissing 2000.

55 Weiß 2000, S. 80.

56 Vgl. Budäus 1998a.
} 
obachter oftmals berechenbarer sind, läßt sich ein erhebliches Steuerungspotential und eine Steuerbarkeit von Netzwerken ausmachen: Sie können sowohl Steuerungssubjekt (Akteur) als auch Steuerungsobjekt sein".57

$\mathrm{Ob}$ die Berechenbarkeitsvermutung stimmt, sei dahingestellt, aber die Parallelität, als Bürger Steuerungssubjekt wie -objekt zu sein, muß nicht mehr als Paradoxie - Bürger als Souverän der Demokratie und gleichzeitig Objekt der Herrschaft von Politik und Bürokratie zu sein - ausgehalten werden.

Demokratie formiert sich als Netzwerk multilateral; Bürger wählen nicht nur, sondern kooperieren untereinander, mit der Politik und der Verwaltung, wobei sich neue Vernetzungsstrukturen ausbilden. eGovernment und eDemocracy sind spezifische Ausprägungen innerhalb dieser Netzwerkstruktur Demokratie.

Das eGovernment kann durch die Unterstützung der Kommunikationstechnologien eine breitere Organisation von zeit- und raumübergreifenden Anspruchsgruppen und damit eine neue Form der Politik hervorbringen: Zum einen kann die Wahrscheinlichkeit eines Organisationsgrades erhöht werden, da die Suche potentieller Mitglieder der Anspruchsgruppen leichter wird. Diese Möglichkeit würde das Konzept gegen erneute Vorwürfe der einfachen Durchsetzung von Partikularinteressen schützen. Zum anderen können durch die kommunalen und regierungsspezifischen Homepages Bürgerbeteiligungsarenen geschaffen werden, in denen eine Transparenz der Argumente produziert wird, die die politische Arena - den politischen Marktplatz der alten Athener - wieder attraktiv macht: eine Agora des digital age: die eGora.

Diese Form der Integration aller möglichen Anspruchsgruppen auf der eGora ist vermutlich auch die einzige Form der politischen Einflußnahme auf die vagabundierenden Netzwerkorganisationen in der Wirtschaft. Die Zivilgesellschaft setzt einerseits die Leistungserbringung wie andererseits auch die Entscheidungsfindung unter Konkurrenz, die nur durch eine nachhaltige Kooperation in einem policy network professionell zu erbringen ist, da nur so die Wünsche und die möglichen Gefahren aufgezeigt werden und der Bürger vor unprofessionellen Eingriffen der Politik bewahrt wird. Diese digital policy networks schaffen Transparenz (neue Öffentlichkeit) und neue demokratische Politikanreize. ${ }^{58}$ Die paradoxe Mischung von Selbststeuerung und dem Primat der Politik wird durch ein eGovernment realistisch.

Immer dort, wo Unklarheit über einen möglichen oder sinnvollen Öffentlichkeitsgrad eines vom Staat zu leistenden Gutes besteht, kann das eGovernment seine e-democracy Dimension ausfalten. Es geht hier nicht um eine formelle Extension demokratischer Wahlverfahren in die mikropolitische Ebene, sondern um tatsächliche Befragungen, Wissenserhebungen, Präferenzeruierungen, Planungsinterventionen. ${ }^{59}$,Die Politik ${ }^{*}$ entscheidet riskante Entscheidungen nicht selber, sondern delegiert sie in solche policy networks, in denen die Interventionsempfänger, partizipatorisch aufgewertet, ihr Wissen mit dem Staat teilen, mit der Chance auf adäquate Interventionen. Die ,alte Politikwelt

57 Weiß 2000, S. 80, mit Bezug auf Mayntz 1993, Mayntz 1990.

${ }^{58} \mathrm{Vgl}$. den Abschnitt, das Netzvolk' in: Leggewie/Maar 1998.

59 Vgl. Slaton 1998, Fishkin 1998. 
erweist sich als überholt", schreibt der niedersächsische Ministerpräsident, es gehe um „stärkere Beteiligung der Menschen am laufenden politischen Planungsprozeß.“60

So entsteht eine neue Kooperationsform des ,knowledge sharing 6 , die in der Form von Bürgerforen, Expertenrunden zum Übernahmegesetz von Unternehmen, Bündnissen für Arbeit etc. längst vor der Durchsetzung der Internet eingeführt wurden. Das klassische Lobbying-Spiel wird nun mit anderer Währung gespielt. Denn auch die Mode des , auditing ${ }^{61}$ darf nicht darüber hinwegtäuschen, daß hier, Clubgüter ' generiert werden, also keine (reinen) öffentlichen Güter. Die Diversifikation der Problemlösungsund , auditing '-Arenen bringt eine ungewohnte Flexibilität in den Politikprozeß, der auf Kosten der Gewährleistung von Allgemeinheit gehen kann. Die Politik muß Instanzen ausbilden, die diese rekursive Schleife im Auge behalten, d.h. interne Interventionssperren ausstellen, die ,die Politik' daran erinnern, daß sie auch als ,Staat ${ }^{6}$ agiert, d.h. als gemeinwohlverpflichtete Institution, die tripartistisch agiert: die Interessen der Exkludierten mit vertretend.

Der Öffentlichkeitsgrad eines Gutes ist nicht nur mit denen, die daran unmittelbar interessiert sind, zu klären, sondern auch mit denen, die unmittelbar nicht betroffen sind. Die Versammlung der unmittelbaren Interventionsempfänger ist eine exklusive Runde zur Erhöhung der Verbindlichkeit wirksamer Politik, aber die Anforderung, die gesamte Wertschöpfungskette des Staates im Auge zu behalten, ist dominant. Ansonsten würde , die Politik ${ }^{\star}$ auf die Lösung von Arena-Problemen retardieren, aber ihre Aufgabe, ,Staat' zu sein, verfehlen. Zwischen Allgemeinheitsanforderung und lokaler oder spezifischer Problemlösungsflexibilität oszilliert die Anforderung staatlicher governanceKompetenz. Wie eingangs beschrieben, kann der schwache oder aktivierende Staat als Moderator zur Beschleunigung und zur ernsthaften Qualifizierung mit seiner eigenen Entscheidung drohen. Der potentielle Wechsel zwischen Aktivierenden und Aktivierten muß bei aller Bescheidenheit gegeben bleiben.

Es wäre vermessen, die eDemocracy als nationale bzw. gar globale Politikinstanz vorzuschlagen. Der vordringlichste Wandel wird lokal geschehen, in den Kommunen. Hier gelten Selbstverpflichtungen aufgrund des Gesetzes des Wiedersehens und die Konsequenzen sind auch offline spürbar. Unabhängig von technischen Möglichkeiten wird die eDomocracy der Kommunen der Prüfstein für ihre neue Effektivität sein. In ihrem Umfeld sind die Bürger kompetent und wagen sich zu äußern, zu intervenieren. Sie wissen, wovon sie reden und können die Handlungspotentiale wie Kommunikationsverläufe einschätzen. So unklug es wäre, nationalwide eDemocracy auszuschließen, so klug ist es, kommunale communites anzubieten. Die Zivilgesellschaft muß selbst erst zivilisiert werden: in Erfahrungsräumen positiver Lernkurven. Diese Entzündungen vor Ort werden zu viralen und viablen Durchdringungen auf Landes- und Bundesebenen führen.

\footnotetext{
60 Gabriel 2000.

61 Vgl. Power 1997.
} 


\section{Literaturverzeichnis}

Barber, B. R. (1998), Which Technology and Which democracy? Democracy and Digital Media, MIT May 8-9, 1998; http//:www.media-in-transition.mit.edu/articles/bar-ber.html

Barzel, Y. (1998), The state and the diversity of third party enforcement. Paper presented at the second annual conference of the International Society of New Institutional Economics, Paris, http//:www. isnie.org/isnie98/Barzel.doc

Berlin Communique (2000), Progressive Governance for the $21^{\text {st }}$ Century. Kommunique der Berliner Konferenz der 14 Staatsmänner, 2.6.2000.

Bimber, B. (1998), The Internet and Political Transformation: Populism, Community and Accelerated Pluralism, in: Polity, Vol. XXXV, No. 1, S. 133-160.

Budäus, D. (Hg., 1998a), Organisationswandel öffentlicher Aufgabenwahrnehmung, Baden-Baden.

Budäus, D. (1998b), Organisationswandel öffentlicher Aufgabenwahrnehmung als Teil eines New Public Management, in: Organisationswandel öffentlicher Aufgabenwahrnehmung, hg. v. D. Budäus, Baden-Baden, S. 99-120.

Cabinet Office (2000), e-government - A Strategic Framework for Public Services in the Information Age, Strategy Paper of the UK „Modernising Government Programme“, April 2000, http//:www.iagchampions.gov.uk/Strategy.htm

Caldow, J. (1998), Governance in the Information Age. A White Paper form the Institute for Electronic Government's $2^{\text {nd }}$ Annual Leadership Workshop, http://www.ieg.ibm.com/pubs/outline.html.

Corrado, A./Firestone, C. M. (Hg., 1996), Elections in the Cyberspace, Washington.

Czada, R./Schmidt, M. G. (Hg., 1993), Verhandlungsdemokratie, Interessenvermittlung, Regierbarkeit. Festschrift für Gerhard Lehmbruch, Opladen.

Deloitte \& Touche (2000), At the Dawn of e-Government. The Citizen as Customer. State Government Approaches to Customer Service. A Public Sector study by Deloitte Consulting and Deloitte \& Touche,http://www.napawash. org/cim. nsf/ac50ab83 963b9499852569 6800 5f 4b 0a/e 137 4d $5720 \mathrm{~d} 8228085256968005 \mathrm{f} 8 \mathrm{dea} / \$ \mathrm{FILE} / \mathrm{At} \sim$ the $\sim$ Dawn of $\sim$ eGovernment.pdf

Dienel, P. C. (1999), Mehr Mitentscheidung in der Demokratie ist möglich, in: Aus Politik und Zeitgeschichte, Bd. 24-25, S. 23-29.

Eggertson, T. (1997), When the State Changes It's Mind: The Puzzle of Discontinuity on Government Control of Economic Activity, in: Privatization at the End of the Century, hg. v. H. Giersch, Berlin/Heidelberg, S. 3-28.

Eisel, S./Scholl, M. (Hg., 1998), Internet und Politik, St. Augustin.

Engel, C./Keller, K. H. (Hg., 2000), Understanding the Impact of Global Networks on Local Social, Political and Cultural Values, Baden-Baden.

Engelhardt, G. (1995), ,Symbiotische Arrangements` und Versorgungsorganisation öffentlicher Aufgabenerfüllung. Diskussionsschriften aus dem Institut für Finanzwissenschaften, Nr. 37, Universität Hamburg.

Fischkin, J. F. (1998), Das ganze Land in einem Raum. Experimente mit beratenden Meinungsumfragen, in: Internet \& Politik - Von der Zuschauer- zur Beteiligungsdemokratie, hg. v. C. Leggewie/C. Maar, Köln, S. 341-353.

Frey, B. S./Kucher, M./Stutzer, A. (1999), Outcome, Process \& Power in Direct Democracy. Working paper No. 25, Institute for Empirical Research in Economics, Universität Zürich.

Fürst, D. (1987), Die Neubelebung der Staatsdiskussion. Veränderte Anforderungen an Regierung und Verwaltung in westlichen Industriegesellschaften, in: Jahrbuch zur Staats- und Verwaltungswissenschaft, Bd. 1, S. 26-53.

Gabriel, S. (2000), Das Ende der Dinosaurier, in: Der Spiegel, 14/2000, S. 40-42.

Gellner, W./Korff, F. v. (Hg., 1998), Demokratie und Internet, Baden-Baden. 
Grossmann, L. (1998), Changing Conceptions of Democracy. Democracy and Digital Media, MIT May 8-9, 1998, http//:www.media-in-transition.mit.edu/articles/grossmann.html.

Hagen, M. (1997), Elektronische Demokratie. Computernetzwerke und politische Theorie in den USA, Hamburg.

Hague, B. N./Loader, B. D. (Hg., 1999), Digital Democracy. Discourse and Decision Making in the Information Sage, London/New York.

Jansen, S. A. (2000), Konkurrenz der Konkurrenz. Co-opetition - Die Form der Konkurrenz - Typen, Funktionen und Voraussetzungen von paradoxen Koordinationsformen, in: Konkurrenz und Kooperation - Interdisziplinäre Beiträge, hg. v. S. A. Jansen/S. Schleissing, Marburg (i. E.).

Jansen, S. A./Priddat, B. P. (2000), http://eGora.gov. From the ,Zivilgesellschaft" to eGovernment: the virtualisation of the state. Paper auf dem Weltkongreß, new governance', 2./3. Juni 2000 Berlin.

Jarren, O. (1998), Demokratie durch Internet?, in: Eisel/Scholl 1998, S. 27-51.

Kahler, M. (2000), Information Networks and Global Politics, in: Understanding the Impact of Global Networks on Local Social, Political and Cultural Values, hg. v. C. Engel/K. H. Keller, BadenBaden, S. 141-157.

Keane, J. (1988), Democracy and Civil Society, London.

Keck, M. E./Sikking, K. (1998), Activist beyond Borders: Advocacy Networks in international Politics, Ithaca/London.

Kenis, P./Schneider, V. (Hg., 1996), Organisation und Netzwerk. Institutionelle Steuerung in Wirtschaft und Politik, Frankfurt/M.

Korff, F. v. (1998), Kommunale Bürgernetze im Internet, in: Demokratie und Internet, hg. v. W. Gellner/F. v. Korff, Baden-Baden, S. 95-110.

Leggewie, C. (1998), Demokratie auf der Datenautobahn - Wie weit geht die Zivilisierung des Cyberspace? Internet und Politik - Ein Problemaufriß, in: Internet \& Politik - Von der Zuschauer- zur Beteiligungsdemokratie, hg. v. C. Leggewie/C. Maar, Köln.

Leggewie, C./Maar, C. (Hg., 1998), Internet \& Politik - Von der Zuschauer- zur Beteiligungsdemokratie, Köln.

Leib, V. (1998), Wissenschaftsnetze und Bürgernetze. Vom selbstgesteuerten Internet zur elektronischen Demokratie?, in: Demokratie und Internet, hg. v. W. Gellner/F. v. Korff, Baden-Baden, S. 81-94.

Magaziner, I. (1998), Democracy and Cyberspace: First Principles; Democracy and Digital media, MIT May 8-9, 1998, http//:www.media-in-transition.mit.edu/articles/session4_ml.html.

March, J. G./Olsen, J. P. (1989), Rediscovering Institutions. The Organizational Basis of Politics, New York/London.

Mayntz, Renate (1990), Politische Steuerbarkeit und Reformblockaden, in: Soziale Dynamik und politische Steuerung, hg. v. R. Mayntz, Frankfurt/M.New York, S. 209-238.

Mayntz, R. (1993), Policy-Netzwerke und die Logik von Verhandlungssystemen, in: Policy-Analyse. Kritik und Neuorientierung, Politische Vierteljahresschrift Sonderheft 24, hg. v. A. Héritier, Opladen, S. 39-56.

McDonough, F. A. (2000), Waiting for e-government, in: Academia. Europäische Akademie Bozen, Nr. 23, S. 8-9.

Mulder, B. (1999), Parliaments Futures: Re-Presenting the Issue. Information, Technology and the Dynamics of Democracy, in: Parliamentary Affairs, Vol. 52, No. 3, S. 553-566.

Noll, W./Ebert, W. (1998), Organisationswandel aus verändertem Verständnis öffentlicher Aufgaben. Geeignete Institutionen aus spieltheoretischer Sicht, in: Organisationswandel öffentlicher Aufgabenwahrnehmung, hg. v. D. Budäus, Baden-Baden, S. 61-98.

Norris, P. (2001), Digital Divide? Civic Engagement, Information Poverty \& the Internet Worldwide, New York, i.E.

Post, D. G./Johnson, D. R. (1997), The New Civic Virtue of the Net: A Complex Systems Model for the Governance of Cyberspace, www.stlr.stanford.edu/STRL/Working_Papers/97_Post_1/index.html. 
Powell, W. W. (1990), Neither Market nor Hierarchy: Netwok Forms of Organizations, in: Research in Organizational Behaviour, Vol. 12, S. 295-304.

Power, M. (1997), The Audit Society. Rituals of Verification, Oxford.

Priddat, B. P. (Hg., 2000), Der bewegte Staat. Formen seiner ReForm; Notizen zur "new governance“, Marburg.

Priddat, B. P. (2000a), ReForm. Über den Wunsch nach Form in der Politik, in: Der bewegte Staat, hg. v. B. P., Marburg, S. 119-166.

Priddat, B. P. (2001), Der Stellenwert korporatistischer Wirtschaftspolitik vor geänderten Rahmenbedingungen, in: Globalisierung, hg. v. C. Smekal/T. Theuerl, i.E.

Resnick, D. (1998), Politics on the Internet. The Normalization of Cyberspace, in: The Politics of Cyberspace, hg. v. C. Toulouse/T. W. Luke, London, S. 48-68.

Ritter, E.-H. (1979), Der kooperative Staat. Bemerkungen zum Verhältnis von Staat und Wirtschaft, in: Archiv des öffentlichen Rechts 104, S. 389-448.

Sassen, S. (2000), The Impact of the Internet on Sovereignty. Unfounded and real Worries, in: Understanding the Impact of Global Networks on Local Social, Political and Cultural Values, hg. v. C. Engel/K. H. Keller, Baden-Baden, S. 189-200.

Scharpf, F. W. (1993), Versuch über Demokratie im verhandelnden Staat, in: Verhandlungsdemokratie, Interessenvermittlung, Regierbarkeit. Festschrift für Gerhard Lehmbruch, R. Czada/M. G. Schmidt, Opladen, S. 25-50.

Schmalz-Bruns, R. (2000), Technokulturelle Visionen zwischen Selbstblockade und Reformstau. Zum demokratischen Potential des Internet (Ms.).

Schudson, M. (1998), Changing Concepts of Democracy. Paper der Konferenz „Democracy and Digital Media“, MIT on May 8-9, 1998 http://media-in-transition.mit.edu/articles/schudson.html.

Schuppert, G. F. (1998), Geändertes Staatsverständnis als Grundlage des Organisationswandels öffentlicher Aufgabenwahrnehmung, in: Organisationswandel öffentlicher Aufgabenwahrnehmung, hg. v. D. Budäus, Baden-Baden, S. 19-60.

Slaton, C. D. (1998), Mündige Bürger durch Televoten. Ein fortlaufendes Experiment zur Transformation der Demokratie, in: Internet \& Politik - Von der Zuschauer- zur Beteiligungsdemokratie, hg. v. C. Leggewie/C. Maar, Köln, S. 312-341.

Stradtmann, P. (1998), Deutschland auf dem Weg in die elektronische Demokratie? Das Internet als neues Medium der politischen Kommunikation und Partizipation. Diplomarbeit an der Hochschule für Musik und Theater Hannover/Institut für Journalistik und Kommunikationsforschung.

Sudgen, R. (1993), A Review of Inequality, re-Examined by Armartya Sen, in: Journal of Economic Literature, Vol. 31, S. 1947-1986.

The White House (2000), Remarks By The President In First Internet Webcast, Office of the Press Secretary, June, 24, 2000.

Wegner, G. (1996), Wirtschaftspolitik zwischen Selbst- und Fremdsteuerung - ein neuer Ansatz, BadenBaden.

Weiß, J. (2000), Umweltpolitik als Akteurshandeln. Eine Theorie der kooperativen Bearbeitung von Informations- und Verteilungsproblemen in der umweltpolitischen Steuerung, Marburg.

Werle, R. (2000), The Impact of Information Networks on the Structure of Political Systems, in: Understanding the Impact of Global Networks on Local Social, Political and Cultural Values, hg. v. C. Engel/K. H. Keller, Baden-Baden, S. 159-185.

Wieland, J. (1999), Die Ethik der Governance, Marburg.

Willke, H. (1992), Ironie des Staates. Grundlinien einer Staatstheorie polyzentrischer Gesellschaft, Frankfurt/M.

Willke, H. (1997), Supervision des Staates, Frankfurt/M.

Zolo, D. (1998), Die demokratische Fürstenherrschaft. Für eine realistische Theorie der Politik, Göttingen. 